\title{
Spectral Properties of Hypoelliptic Operators
}

\author{
J.-P. Eckmann ${ }^{1,2}$ and M. Hairer ${ }^{1}$ \\ 1 Département de Physique Théorique, Université de Genève \\ 2 Section de Mathématiques, Université de Genève \\ Email: Jean-Pierre.Eckmann@physics.unige.ch \\ Email: Martin.Hairer@physics.unige.ch
}

\begin{abstract}
We study hypoelliptic operators with polynomially bounded coefficients that are of the form $K=\sum_{i=1}^{m} X_{i}^{T} X_{i}+X_{0}+f$, where the $X_{j}$ denote first order differential operators, $f$ is a function with at most polynomial growth, and $X_{i}^{T}$ denotes the formal adjoint of $X_{i}$ in $\mathrm{L}^{2}$. For any $\varepsilon>0$ we show that an inequality of the form $\|u\|_{\delta, \delta} \leq$ $C\left(\|u\|_{0, \varepsilon}+\|(K+i y) u\|_{0,0}\right)$ holds for suitable $\delta$ and $C$ which are independent of $y \in \mathbf{R}$, in weighted Sobolev spaces (the first index is the derivative, and the second the growth). We apply this result to the Fokker-Planck operator for an anharmonic chain of oscillators coupled to two heat baths. Using a method of Hérau and Nier [HNO2], we conclude that its spectrum lies in a cusp $\left\{x+\left.i y|x \geq| y\right|^{\tau}-c, \tau \in(0,1], c \in \mathbf{R}\right\}$.
\end{abstract}

\section{Introduction}

In an interesting paper, [HNO2], Hérau and Nier studied the Fokker-Planck equation associated to a Hamiltonian system $H$ in contact with a heat reservoir at inverse temperature $\beta$. For this problem, it is well-known that the Gibbs measure

$$
\mu_{\beta}(d p d q)=\exp (-\beta H(p, q)) d p d q
$$

is the only invariant measure for the system. In their study of convergence under the flow of any measure to the invariant measure, they were led to study spectral properties of the Fokker-Planck operator $\mathcal{L}$ when considered as an operator on $\mathrm{L}^{2}\left(\mu_{\beta}\right)$. In particular, they showed that $\mathcal{L}$ has a compact resolvent and that its spectrum is located in a cusp-shaped region, as depicted in Figure 1 below, improving (for a special case) earlier results obtained by Rey-Bellet and Thomas [RBT02b], who showed that $e^{-\mathcal{L} t}$ is compact and that $\mathcal{L}$ has spectrum only in $\operatorname{Re} \lambda>\mathrm{c}>0$ aside from a simple eigenvalue at 0 .

Extending the methods of [HN02], we show in this paper that the cusp-shape of the spectrum of $\mathcal{L}$ occurs for many Hörmander-type operators of the form

$$
K=\sum_{i=1}^{m} X_{i}^{T} X_{i}+X_{0}+f,
$$




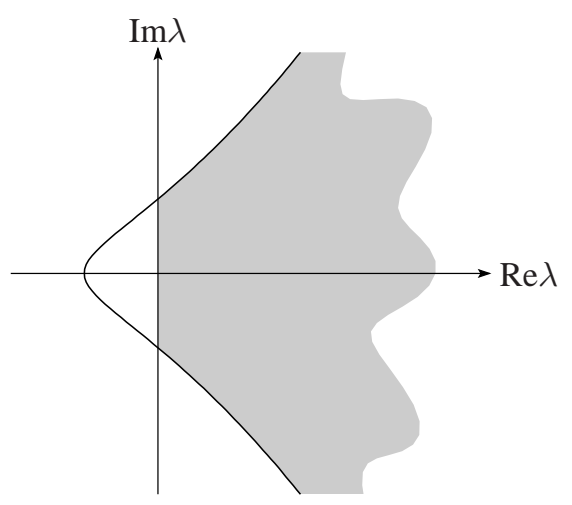

Figure 1 Cusp containing the spectrum of $\mathcal{L}$.

(the symbol ${ }^{T}$ denotes the formal adjoint in $\mathrm{L}^{2}$ ) when the family of vector fields $\left\{X_{j}\right\}_{j=0}^{m}$ is sufficiently non-degenerate (see Definition 2.1 and assumption $b_{1}$ below) and some growth condition on $f$ holds.

The main motivation for our paper comes from the study of the model of heat conduction proposed in [EPR99a] and further studied in [EPR99b, EH00, RBT00, RBT02b, RBT02a]. These papers deal with Hamiltonian anharmonic chains of pointlike particles with nearest-neighbor interactions whose ends are coupled to heat reservoirs modeled by linear classical field theories. Our results improve the detailed knowledge about the spectrum of the generator $\mathcal{L}$ of the associated Markov process, see Sect. 5. As a by-product, our paper also gives a more elegant analytic proof of the results obtained in [EH00]. A short probabilistic proof has already been obtained in [RBT02b].

The main technical result needed to establish the cusp-form of the spectrum is the Sobolev estimate Theorem 4.1 which seems to be new.

\section{Acknowledgements}

We thank G. van Baalen and E. Zabey for helpful remarks. This work was partially supported by the Fonds National Suisse.

\section{Setup and Notations}

We will derive lower bounds for hypoelliptic operators with polynomially bounded coefficients that are of the form (1.1). We start by defining the class of functions and vector fields we consider.

\subsection{Notations}

For $N \in \mathbf{R}$, we define the set $\mathbf{P o l}_{0}^{N}$ of polynomially growing functions by

$$
\mathbf{P o l}_{0}^{N}=\left\{f \in \mathcal{C}^{\infty}\left(\mathbf{R}^{n}\right) \mid \forall \alpha, \sup _{x \in \mathbf{R}^{n}}(1+\|x\|)^{-N} \partial^{\alpha} f(x) \leq C_{\alpha}\right\} .
$$


In this expression, $\alpha$ denotes a multi-index of arbitrary order. We also define the set $\mathbf{P o l}_{1}^{N}$ of vector fields in $\mathbf{R}^{n}$ that can be written as

$$
G=G_{0}(x)+\sum_{j=1}^{n} G_{j}(x) \partial_{j}, \quad G_{i} \in \mathbf{P o l}_{0}^{N}
$$

One can similarly define sets $\mathbf{P o l}_{k}^{N}$ of $k$ th order differential operators. It is clear that if $X \in \mathbf{P o l}_{k}^{N}$ and $Y \in \mathbf{P o l}_{\ell}^{M}$, then $[X, Y] \in \mathbf{P o l}_{k+\ell-1}^{N+M}$. If $f$ is in $\mathbf{P o l}_{0}^{N}$, but not in $\mathbf{P o l}_{0}^{N+\varepsilon}$ for any $\varepsilon>0$, we say it is of degree $N$.

\subsection{Hypotheses}

Definition 2.1 A family $\left\{A_{i}\right\}_{i=1}^{m}$ of vector fields in $\mathbf{R}^{n}$ with $A_{i}=\sum_{j=1}^{n} A_{i, j} \partial_{j}$ is called non-degenerate if there exist constants $N$ and $C$ such that for every $x \in \mathbf{R}^{n}$ and every vector $v \in \mathbf{R}^{n}$ one has the bound

$$
\|v\|^{2} \leq C\left(1+\|x\|^{2}\right)^{N} \sum_{i=1}^{m}\left\langle A_{i}(x), v\right\rangle^{2},
$$

with $\left\langle A_{i}(x), v\right\rangle=\sum_{j=1}^{n} A_{i, j}(x) v_{j}$.

The conditions on $K$ which we will use below are taken from the following list.

$a$. The vector fields $X_{j}$ with $j=0, \ldots, m$ belong to $\mathbf{P o l}_{1}^{N}$ and the function $f$ belongs to $\mathbf{P o l}_{0}^{N}$.

$b_{0}$. There exists a finite number $M$ such that the family consisting of $\left\{X_{i}\right\}_{i=0}^{m}$, $\left\{\left[X_{i}, X_{j}\right]\right\}_{i, j=0}^{m},\left\{\left[\left[X_{i}, X_{j}\right], X_{k}\right]\right\}_{i, j, k=0}^{m}$ and so on up to commutators of rank $M$ is non-degenerate.

$b_{1}$. There exists a finite number $M$ such that the family consisting of $\left\{X_{i}\right\}_{i=1}^{m}$, $\left\{\left[X_{i}, X_{j}\right]\right\}_{i, j=0}^{m},\left\{\left[\left[X_{i}, X_{j}\right], X_{k}\right]\right\}_{i, j, k=0}^{m}$ and so on up to commutators of rank $M$ is non-degenerate.

The difference between $b_{0}$ and $b_{1}$ is in the inclusion of the vector field $X_{0}$ (in $b_{0}$ ), so that $b_{1}$ is stronger than $b_{0}$.

Definition 2.2 We call $\mathcal{K}_{0}$ the class of operators of the form of (1.1) satisfying a and $b_{0}$ above, and $\mathcal{K}_{1}$ the class of those satisfying a and $b_{1}$. Clearly, $b_{1}$ is more restrictive than $b_{0}$ and therefore $\mathcal{K}_{1} \subset \mathcal{K}_{0}$.

Remark 2.3 If $K$ is in $\mathcal{K}_{0}$ then $K$ is hypoelliptic. If $K$ is in $\mathcal{K}_{1}$ then $\partial_{t}+K$ is hypoelliptic. 


\section{Localized Bound}

The main result of this section is Theorem 3.1 which provides bounds for localized test functions.

We let $\mathcal{B}(x)$ denote the unit cube around $x \in \mathbf{R}^{n}$ :

$$
\mathcal{B}(x)=\left\{y \in \mathbf{R}^{n}|| y_{j}-x_{j} \mid \leq 1, j=1, \ldots, n\right\} .
$$

To formulate our bounds, we introduce the operator $\Lambda$, defined as the positive square root of $\Lambda^{2}=1-\sum_{i=1}^{n} \partial_{i}^{2}=1-\Delta$. Later on, we will also need the multiplication operator $\bar{\Lambda}$ defined as the positive root of (multiplication by) $\bar{\Lambda}^{2}=1+\|x\|^{2}$.

Theorem 3.1 Assume $K \in \mathcal{K}_{1}$. Then, there exist positive constants $\varepsilon_{*}, C_{*}$, and $N_{*}$ such that for every $x \in \mathbf{R}^{n}$ and every $u \in \mathcal{C}_{0}^{\infty}(\mathcal{B}(x))$, one has uniformly for $y \in \mathbf{R}^{n}$ :

$$
\left\|\Lambda^{\varepsilon_{*}} u\right\| \leq C_{*}\left(1+\|x\|^{2}\right)^{N_{*}}\|u\|+\|(K+i y) u\| .
$$

If $K$ is in $\mathcal{K}_{0}$ (but not in $\mathcal{K}_{1}$ ) the same estimate holds, but the constant $C_{*}$ will depend generally on $y{ }^{1}$

Proof. The novelty of the bound is in allowing for polynomial growth of the coefficients of the differential operators. Were it not for this, the result would be a special case of Hörmander's proof of hypoellipticity of second-order partial differential operators [Hör85, Thm. 22.2.1]. Since the coefficients of our differential operators can grow polynomially we need to work with weighted spaces.

We introduce a family of weighted Sobolev spaces $S^{\alpha, \beta}$ with $\alpha, \beta \in \mathbf{R}$ as the following subset of tempered distributions $\mathrm{S}_{n}^{\prime}$ on $\mathbf{R}^{n}$ :

$$
S^{\alpha, \beta}=\left\{u \in \mathrm{S}_{n}^{\prime} \mid \Lambda^{\alpha} \bar{\Lambda}^{\beta} u \in \mathrm{L}^{2}\left(\mathbf{R}^{n}\right)\right\} .
$$

We equip this space with the scalar product

$$
\langle f, g\rangle_{\alpha, \beta}=\left\langle\Lambda^{\alpha} \bar{\Lambda}^{\beta} f, \Lambda^{\alpha} \bar{\Lambda}^{\beta} g\right\rangle_{\mathrm{L}^{2}},
$$

writing also $\langle\cdot, \cdot\rangle_{\alpha}$ instead of $\langle\cdot, \cdot\rangle_{\alpha, 0}$. We also use the corresponding norms $\|\cdot\|_{\alpha, \beta}$. Note that these spaces are actually a particular case of the more general class of Sobolev spaces introduced in [BC94].

The following lemma lists a few properties of the spaces $S^{\alpha, \beta}$ that will be useful in the sequel. We postpone its proof to Appendix A.

Lemma 3.2 Let $\alpha, \beta \in \mathbf{R}$. We have the following:

a. Embedding: For $\alpha^{\prime} \geq \alpha$ and $\beta^{\prime} \geq \beta$, the space $S^{\alpha^{\prime}, \beta^{\prime}}$ is continuously embedded into $S^{\alpha, \beta}$. The embedding is compact if and only if both inequalities are strict.

b. Scales of spaces: The operators $\Lambda^{\gamma}$ and $\bar{\Lambda}^{\gamma}$ are bounded from $S^{\alpha, \beta}$ into $S^{\alpha-\gamma, \beta}$ and $S^{\alpha, \beta-\gamma}$ respectively. If $X \in \mathbf{P o l}_{k}^{N}$ then $X$ is bounded from $S^{\alpha, \beta}$ into $S^{\alpha-k, \beta-N}$.

\footnotetext{
${ }^{1}$ The norms are $\mathrm{L}^{2}$ norms.
} 
c. Polarization: For every $\gamma, \delta \in \mathbf{R}$, one has the bound

$$
\langle f, g\rangle_{\alpha, \beta} \leq C\|f\|_{\alpha^{\prime}, \beta^{\prime}}\|g\|_{\alpha^{\prime \prime}, \beta^{\prime \prime}}, \quad \alpha^{\prime}+\alpha^{\prime \prime}=2 \alpha, \beta^{\prime}+\beta^{\prime \prime}=2 \beta,
$$

which holds for all $f$ and $g$ belonging to the Schwartz space $\mathrm{S}_{n}$. The constant $C$ may depend on the indices.

d. Commutator: Let $X \in \mathbf{P o l}_{k}^{N}$ and $Y \in \mathbf{P o l}_{k^{\prime}}^{N^{\prime}}$. For every $\gamma \in \mathbf{R},\left[X, \Lambda^{\gamma}\right]$ is bounded from $S^{\alpha, \beta}$ into $S^{\alpha+1-k-\gamma, \beta-N}$. Similarly, $\left[X,\left[Y, \Lambda^{\gamma}\right]\right]$ is bounded from $S^{\alpha, \beta}$ into $S^{\alpha+2-k-k^{\prime}-\gamma, \beta-N-N^{\prime}}$.

e. Adjoint: Let $X \in \mathbf{P o l}_{k}^{N}$ and let $f, g \in \mathrm{S}_{n}$. Then

$$
\langle f, X g\rangle_{\alpha, \beta}=\left\langle X^{T} f, g\right\rangle_{\alpha, \beta}+R(f, g),
$$

where the bilinear form $R$ satisfies the bound

$$
|R(f, g)| \leq C\|f\|_{\alpha^{\prime}, \beta^{\prime}}\|g\|_{\alpha^{\prime \prime}, \beta^{\prime \prime}},
$$

with

$$
\alpha^{\prime}+\alpha^{\prime \prime}=2 \alpha+k-1, \quad \beta^{\prime}+\beta^{\prime \prime}=2 \beta+N .
$$

The constant $C$ may depend on the indices.

Notation 3.3 We write $K_{y}$ instead of $K+i y$. We also introduce the notation $\Phi \leq \mathrm{B}$ to mean: There exist constants $C$ and $N$ independent of $x$ and $y$ such that for all $u \in \mathcal{C}_{0}^{\infty}(\mathcal{B}(x))$ :

$$
\Phi \leq C(1+\|x\|)^{N}\left(\|u\|+\left\|K_{y} u\right\|\right) .
$$

We will show below that

$$
\left\|A \Lambda^{\varepsilon-1} u\right\| \leq \mathrm{B}
$$

holds for $A$ taking values among all of the vector fields appearing in $b_{1}$ or $b_{0}$. Assuming (3.4) one completes the proof of Theorem 3.1 as follows: Notice that if the collection $\left\{A_{i}\right\}_{i=1}^{k}$ is non-degenerate, then

$$
\|\Lambda u\|^{2} \leq C_{1}\left(1+\|x\|^{2}\right)^{N_{1}} \sum_{i=1}^{k}\left\|A_{i} u\right\|^{2},
$$

for every $x \in \mathbf{R}^{n}$ and every $u \in \mathcal{C}_{0}^{\infty}(\mathcal{B}(x))$. Therefore, by (3.4) we find

$$
\|u\|_{\varepsilon}^{2}=\left\|\Lambda \Lambda^{\varepsilon-1} u\right\| \leq C_{1}\left(1+\|x\|^{2}\right)^{N_{1}} \sum_{i=1}^{k}\left\|A_{i} \Lambda^{\varepsilon-1} u\right\|^{2} \leq \mathrm{B}^{2} .
$$

Polarizing, we obtain:

$$
\begin{aligned}
\|u\|_{\varepsilon / 2}^{2} & \leq\|u\|\|u\|_{\varepsilon} \leq C_{2}\|u\|\left(1+\|x\|^{2}\right)^{N_{2}}\left(\|u\|+\left\|K_{y} u\right\|\right) \\
& \leq C_{2}^{2}\|u\|^{2}\left(1+\|x\|^{2}\right)^{2 N_{2}}+\left(\|u\|+\left\|K_{y} u\right\|\right)^{2} \\
& \leq\left(C_{2}\|u\|\left(1+\|x\|^{2}\right)^{N_{2}}+\|u\|+\left\|K_{y} u\right\|\right)^{2}
\end{aligned}
$$

and hence (3.1) follows with $\varepsilon_{*}=\varepsilon / 2, N_{*}=N_{2}$, and $C_{*}=C_{2}+1$.

It remains to prove (3.4). 
Remark 3.4 To the end of this proof, we use the symbols $C$ and $N$ to denote generic positive constants which may change from one inequality to the next.

By the bound on $\left[A, \Lambda^{\varepsilon-1}\right]$ of Lemma 3.2(d) - and the fact that $u \in \mathcal{C}_{0}^{\infty}(\mathcal{B}(x))$ implies $\|u\|_{0, N} \leq C\left(1+\|x\|^{2}\right)^{N / 2}\|u\|$ for every $N>0$-we will have shown (3.4) if we can prove

$$
\|A u\|_{\varepsilon-1} \leq \mathrm{B} .
$$

Notice that by Lemma 3.2(b), the estimate (3.5) yields

$$
\|A u\|_{\varepsilon-1, \gamma}^{2} \leq C_{\gamma}\left(1+\|x\|^{2}\right)^{\gamma+N}\left(\|u\|^{2}+\left\|K_{y} u\right\|^{2}\right),
$$

for every $\gamma>0, x \in \mathbf{R}^{n}$, and $u \in \mathcal{C}_{0}^{\infty}(\mathcal{B}(x))$.

To prove (3.5), we proceed as follows. First, we verify it for $A=X_{i}$ with $i=$ $1, \ldots, m$ (as well as for $A=X_{0}$ in the case $\mathcal{K}_{0}$ ). The remaining bounds are shown by induction. The induction step consists in proving that if (3.5) holds for some $A \in \mathbf{P o l}_{1}^{N}$ then

$$
\left\|\left[A, X_{i}\right] u\right\|_{\varepsilon / 8-1} \leq \mathrm{B} \text { for } i=0, \ldots, m .
$$

The first step. By the definition of $K$ and the fact that $X_{i}$ maps $\mathcal{C}_{0}^{\infty}(\mathcal{B}(x))$ into itself, we see that

$$
\left\|X_{i} u\right\| \leq \mathrm{B}, \quad i=1, \ldots, m,
$$

that is, (3.5) holds for $\varepsilon \leq 1$ and $A=X_{i}$.

We next show that it also holds for $A=X_{0}$ whenever $\varepsilon \leq 1 / 2$. (This will be the only place in the proof where $C$ depends on $y$, but we need this estimate only for the case $\mathcal{K}_{0}$.) Using (1.1) and Lemma 3.2(c), we can write

$$
\left\|X_{0} u\right\|_{-1 / 2}^{2} \leq\left\|X_{0} u\right\|_{-1}\left(\left\|K_{y} u\right\|+\|f u\|+|y|\|u\|\right)+\sum_{i=1}^{m}\left\langle X_{0} u, X_{i}^{T} X_{i} u\right\rangle_{-1 / 2} .
$$

Using Lemma 3.2(b) to estimate $\left\|X_{0} u\right\|_{-1}$, the first term is bounded by $\mathrm{B}^{2}$, so it remains to bound $\left\langle X_{0} u, X_{i}^{T} X_{i} u\right\rangle_{-1 / 2}$. Using this time Lemma 3.2(e), (with $\alpha=-\frac{1}{2}$ and $\beta=0$ ), we write

$$
\left\langle X_{0} u, X_{i}^{T} X_{i} u\right\rangle_{-1 / 2}=\left\langle X_{i} X_{0} u, X_{i} u\right\rangle_{-1 / 2}+R\left(X_{0} u, X_{i} u\right),
$$

where $R\left(X_{0} u, X_{i} u\right)$ is bounded by $C\left\|X_{0} u\right\|_{-1}\left\|X_{i} u\right\|$, which in turn is bounded by $\mathrm{B}^{2}$, using the previous bounds on $\left\|X_{0} u\right\|_{-1}$ and $\left\|X_{i} u\right\|$. The first term of (3.9) can be written as

$$
\left|\left\langle X_{i} X_{0} u, X_{i} u\right\rangle_{-1 / 2}\right| \leq C\left\|X_{i} X_{0} u\right\|_{-1}\left\|X_{i} u\right\| \text {. }
$$

Since $\left\|X_{i} u\right\| \leq \mathrm{B}$ by (3.8), we only need to bound $\left\|X_{i} X_{0} u\right\|_{-1}$ by $\mathrm{B}$. This is achieved by writing

$$
\left\|X_{i} X_{0} u\right\|_{-1} \leq\left\|X_{0} X_{i} u\right\|_{-1}+\left\|\left[X_{i}, X_{0}\right] u\right\|_{-1} .
$$

The second term is bounded by B using Lemma 3.2(b). The first term is also bounded by B since $\left\|X_{i} u\right\|_{0, N} \leq C(1+\|x\|)^{N}\left\|X_{i} u\right\|$ and $X_{0}$ is bounded from $S^{0, N}$ into $S^{-1,0}$ (for some $N$ ) by Lemma 3.2(b). Therefore, we conclude that

$$
\left\|X_{0} u\right\|_{-1 / 2} \leq \mathrm{B}
$$


where $C$ will in general depend on $y$.

The inductive step. Let $A \in \mathbf{P o l}_{1}^{N}$ and assume that (3.5) holds. We show that a similar estimate (with different values for $\varepsilon, C$, and $N$ ) then also holds for $B=\left[A, X_{i}\right]$ with $i=0, \ldots, m$. We distinguish the case $i=0$ from the others.

The case $\boldsymbol{i}>\boldsymbol{0}$. We assume that (3.5) holds and we estimate $\|B u\|_{\varepsilon^{\prime}-1}$ for some $\varepsilon^{\prime} \leq 1 / 2$ to be fixed later. We obtain

$$
\|B u\|_{\varepsilon^{\prime}-1}^{2}=\left\langle B u, A X_{i} u\right\rangle_{\varepsilon^{\prime}-1}-\left\langle B u, X_{i} A u\right\rangle_{\varepsilon^{\prime}-1}=T_{1}+T_{2}
$$

Both terms $T_{1}$ and $T_{2}$ are estimated separately. For $T_{1}$, we get from Lemma 3.2(e):

$$
T_{1}=-\left\langle A B u, X_{i} u\right\rangle_{\varepsilon^{\prime}-1}+R\left(B u, X_{i} u\right),
$$

where (since $\left.\varepsilon^{\prime} \leq 1 / 2\right)$,

$$
\left|R\left(B u, X_{i} u\right)\right| \leq C(1+\|x\|)^{N}\|B u\|_{-1}\left\|X_{i} u\right\| \leq C(1+\|x\|)^{N}\|u\|\left\|X_{i} u\right\| \leq \mathrm{B}^{2} .
$$

The term $\left\langle A B u, X_{i} u\right\rangle_{\varepsilon^{\prime}-1}$ is written as

$$
\left|\left\langle A B u, X_{i} u\right\rangle_{\varepsilon^{\prime}-1}\right| \leq\|B A u\|_{2 \varepsilon^{\prime}-2}\left\|X_{i} u\right\|+\|[A, B] u\|_{-1}\left\|X_{i} u\right\| .
$$

The second term is bounded by $\mathrm{B}^{2}$ like in (3.11). The first term is also bounded by $\mathrm{B}^{2}$ by combining Lemma 3.2(b) with the induction assumption in its form (3.6) (taking $\left.2 \varepsilon^{\prime} \leq \varepsilon\right)$. The estimation of $T_{2}$ is very similar: we write again

$$
T_{2}=-\left\langle X_{i} B u, A u\right\rangle_{\varepsilon^{\prime}-1}+R(B u, A u) .
$$

The first term is bounded by $C\left\|X_{i} B u\right\|_{-1}\|A u\|_{2 \varepsilon^{\prime}-1}$. The second factor of this quantity is bounded by B by the inductive assumption, while the first factor is bounded by

$$
\left\|X_{i} B u\right\|_{-1} \leq\left\|B X_{i} u\right\|_{-1}+\left\|\left[B, X_{i}\right] u\right\|_{-1} \leq \mathrm{B},
$$

using Lemma 3.2(b) and the estimate $\left\|X_{i}\right\|_{0, N} \leq \mathrm{B}$. The remainder $R$ of (3.12) is bounded by

$$
|R(B u, A u)| \leq\|B u\|_{-1}\|A u\|_{2 \varepsilon^{\prime}-1},
$$

which is bounded by $\mathrm{B}^{2}$, using Lemma 3.2(b) for the first factor and the inductive assumption for the second. Combining the estimates on $T_{1}$ and $T_{2}$ we get

$$
\|B u\|_{\varepsilon^{\prime}-1} \leq \mathrm{B} \quad \text { for } \quad \varepsilon^{\prime} \leq \varepsilon / 2
$$

which is the required estimate.

The case $\boldsymbol{i}=\boldsymbol{0}$. To conclude the proof of Theorem 3.1, it remains to bound $\|B u\|_{\varepsilon^{\prime}-1}$ by $\mathrm{B}$. In this expression, $B=\left[A, X_{0}\right]$ and $\varepsilon^{\prime}>0$ is to be fixed later. We first introduce the operator

$$
\tilde{K}=\sum_{i=1}^{m} X_{i}^{T} X_{i},
$$


which is (up to a term of multiplication by a function) equal to the real part of $K_{y}$, when considered as an operator on $\mathrm{L}^{2}$. We can thus write $X_{0}$ as

$$
X_{0}=K-\tilde{K}+f_{1}=\tilde{K}-K^{T}+f_{2}
$$

for two functions $f_{1}, f_{2} \in \mathbf{P o l}_{0}^{N}$ for some $N$. This allows us to express $B$ as

$$
B=\left[A, X_{0}\right]=A K_{y}+K_{y}^{T} A+[\tilde{K}, A]-2 \tilde{K} A+A f_{1}-f_{2} A
$$

We write $\|B u\|_{\varepsilon^{\prime}-1}^{2}=\left\langle B u,\left[A, X_{0}\right] u\right\rangle_{\varepsilon^{\prime}-1}$ and we bound separately by $\mathrm{B}^{2}$ each of the terms that appear in this expression according to the above decomposition of the commutator.

The two terms containing $f_{1}$ and $f_{2}$ are bounded by $\mathrm{B}^{2}$ using the inductive assumption. We therefore concentrate on the four remaining terms.

The term $\boldsymbol{A} \boldsymbol{K}_{\boldsymbol{y}}$. We write this term as

$$
\left\langle B u, A K_{y} u\right\rangle_{\varepsilon^{\prime}-1}=-\left\langle B A u, K_{y} u\right\rangle_{\varepsilon^{\prime}-1}+\left\langle[A, B] u, K_{y} u\right\rangle_{\varepsilon^{\prime}-1}+R\left(B u, K_{y} u\right),
$$

where the two last terms are bounded by $\mathrm{B}^{2}$ using Lemma 3.2(b,e). Using assumption (3.6) (assuming $\varepsilon^{\prime} \leq \varepsilon / 2$ ) and Lemma 3.2(b,c), we also bound the first term by $\mathrm{B}^{2}$.

The $\operatorname{term} \boldsymbol{K}_{\boldsymbol{y}}^{\boldsymbol{T}} \boldsymbol{A}$. We write this term as

$$
\left\langle B u, K_{y}^{T} A\right\rangle_{\varepsilon^{\prime}-1}=\left\langle K_{y} B u, A\right\rangle_{\varepsilon^{\prime}-1}+\left\langle\Lambda^{2-2 \varepsilon^{\prime}}\left[K, \Lambda^{2 \varepsilon^{\prime}-2}\right] B u, A u\right\rangle_{\varepsilon^{\prime}-1}=T_{1}+T_{2} .
$$

The term $T_{1}$ is bounded by $\left\|K_{y} B u\right\|_{-1}\|A u\|_{2 \varepsilon^{\prime}-1}$ by polarization. The second factor of this product is bounded by $\mathrm{B}$, using the induction hypothesis and the assumption $\varepsilon^{\prime} \leq \varepsilon / 2$. The first factor is bounded by

$$
\left\|K_{y} B u\right\|_{-1} \leq\left\|B K_{y} u\right\|_{-1}+\|[K, B] u\|_{-1} .
$$

The first term of this sum is obviously bounded by B. The second term is expanded using the explicit form of $K$ as given in (1.1). The only "dangerous" terms appearing in this expansion are those of the form $\left\|\left[X_{i}^{T} X_{i}, B\right] u\right\|_{-1}$. They are bounded by

$$
\left\|\left[X_{i}^{T} X_{i}, B\right] u\right\|_{-1} \leq\left\|\left[X_{i}^{T}, B\right] X_{i} u\right\|_{-1}+\left\|\left[X_{i}, B\right] X_{i}^{T} u\right\|_{-1}+\left\|\left[X_{i}^{T},\left[X_{i}, B\right]\right] u\right\|_{-1} .
$$

The terms in this sum are bounded individually by B, using the estimates on $\left\|X_{i} u\right\|$, together with Lemma 3.2(b,d). We now turn to the term $T_{2}$. We bound it by

$$
\left|T_{2}\right| \leq C\left\|\Lambda^{2-2 \varepsilon^{\prime}}\left[K, \Lambda^{2 \varepsilon^{\prime}-2}\right] B u\right\|_{-1}\|A u\|_{2 \varepsilon^{\prime}-1} .
$$

The second factor is bounded by B by the induction hypothesis, so we focus on the first factor. We again write explicitly $K$ as in (1.1) and estimate each term separately. The two terms containing $X_{0}$ and $f$ are easily bounded by B using Lemma 3.2(b,d). We also write $X_{i}^{T} X_{i}=X_{i}^{2}+Y_{i}$ with $Y_{i} \in \mathbf{P o l}_{1}^{N}$ and similarly bound by B the terms in $Y_{i}$. The remaining terms are of the type

$$
Q_{i}=\left\|\Lambda^{2-2 \varepsilon^{\prime}}\left[X_{i}^{2}, \Lambda^{2 \varepsilon^{\prime}-2}\right] B u\right\|_{-1}
$$


They are bounded by

$$
Q_{i} \leq 2\left\|\Lambda^{2-2 \varepsilon^{\prime}}\left[X_{i}, \Lambda^{2 \varepsilon^{\prime}-2}\right] X_{i} B u\right\|_{-1}+\left\|\Lambda^{2-2 \varepsilon^{\prime}}\left[X_{i},\left[X_{i}, \Lambda^{2 \varepsilon^{\prime}-2}\right]\right] B u\right\|_{-1} .
$$

In order to bound the first term, one writes $X_{i} B=B X_{i}+\left[X_{i}, B\right]$ and bounds each term separately by $\mathrm{B}$, using the bound $\left\|X_{i} u\right\|_{0, \gamma} \leq \mathrm{B}$ together with Lemma 3.2(b,d). The last term is also bounded by B using Lemma 3.2(d).

The term $[\tilde{\boldsymbol{K}}, \boldsymbol{A}]$. We write $\tilde{K}=\sum_{i=1}^{m} X_{i}^{T} X_{i}$ and we bound each term separately:

$$
\begin{aligned}
\left\langle B u,\left[X_{i}^{T} X_{i}, A\right] u\right\rangle_{\varepsilon^{\prime}-1} & =\left\langle B u, X_{i}^{T}\left[X_{i}, A\right] u\right\rangle_{\varepsilon^{\prime}-1}+\left\langle B u,\left[X_{i}^{T}, A\right] X_{i} u\right\rangle_{\varepsilon^{\prime}-1} \\
& \equiv T_{i, 1}+T_{i, 2} .
\end{aligned}
$$

The first term is written as

$$
T_{i, 1}=\left\langle X_{i} B u,\left[X_{i}, A\right] u\right\rangle_{\varepsilon^{\prime}-1}+R(u),
$$

where $R(u)$ is bounded by $C\|B u\|_{-1}\left\|\left[X_{i}, A\right] u\right\|_{2 \varepsilon^{\prime}-1}$. The first factor is bounded by B using Lemma 3.2(b) and the second factor is bounded by B, using the estimate for the case $i \neq 0$ (we have to assume $\varepsilon^{\prime} \leq \varepsilon / 4$ in order to get this bound). The term $\left\langle X_{i} B u,\left[X_{i}, A\right] u\right\rangle_{\varepsilon^{\prime}-1}$ is estimated by

$$
\left|\left\langle X_{i} B u,\left[X_{i}, A\right] u\right\rangle_{\varepsilon^{\prime}-1}\right| \leq\left\|X_{i} B u\right\|_{-1}\left\|\left[X_{i}, A\right] u\right\|_{2 \varepsilon^{\prime}-1} .
$$

The first factor is bounded by $\mathrm{B}$ as in (3.13) and the second factor is again bounded by $\mathrm{B}$, using the estimate for the case $i \neq 0$. It thus remains to bound $T_{i, 2}$, which we write as

$$
T_{i, 2}=\left\langle B u, X_{i}\left[X_{i}^{T}, A\right] u\right\rangle_{\varepsilon^{\prime}-1}+\left\langle B u,\left[\left[X_{i}^{T}, A\right], X_{i}\right] u\right\rangle_{\varepsilon^{\prime}-1} .
$$

The first term in this equation is similar to the term $\left\langle B u, X_{i}^{T}\left[X_{i}, A\right] u\right\rangle_{\varepsilon^{\prime}-1}$ and is bounded by $\mathrm{B}^{2}$ in the same way. The second term is bounded by

$$
\left\langle B u,\left[\left[X_{i}^{T}, A\right], X_{i}\right] u\right\rangle_{\varepsilon^{\prime}-1} \leq\|B u\|_{-1}\left\|\left[\left[X_{i}^{T}, A\right], X_{i}\right] u\right\|_{2 \varepsilon^{\prime}-1},
$$

which can also be bounded by $\mathrm{B}^{2}$, using the estimate for the case $i \neq 0$, provided $\varepsilon^{\prime} \leq \varepsilon / 8$.

The term $\tilde{\boldsymbol{K}} \boldsymbol{A}$. In order to bound this term, we need the following preliminary lemma:

Lemma 3.5 Let $v \in \mathrm{S}_{n}, \alpha, \delta \in \mathbf{R}$, and let $K_{y}$ be as above. There exist constants $\tilde{C}$ and $\tilde{N}$ independent of $y$ such that the estimate

$$
\left|\operatorname{Re}\left\langle K_{y} v, v\right\rangle_{\alpha}-\sum_{i=1}^{m}\left\|X_{i} v\right\|_{\alpha}^{2}\right| \leq \tilde{C} \sum_{i=1}^{m}\left\|X_{i} v\right\|_{\alpha-\delta, \tilde{N}}\|v\|_{\alpha+\delta, \tilde{N}}+\tilde{C}\|v\|_{\alpha, \tilde{N}}^{2}
$$

holds.

Proof. Obviously $\left\langle K_{y} v, v\right\rangle_{\alpha}=\langle K v, v\rangle_{\alpha}$. We decompose $K$ according to (1.1). The terms containing $X_{0}$ and $f$ are bounded by $C\|v\|_{\alpha, N}^{2}$ according to Lemma 3.2(b,e), so we focus on the terms containing $X_{i}^{T} X_{i}$. Using Lemma 3.2(e), we write them as

$$
\left\langle X_{i}^{T} X_{i} v, v\right\rangle_{\alpha}=\left\|X_{i} v\right\|_{\alpha}^{2}+R_{i}(v),
$$

where $R_{i}(v)$ is bounded by $C\left\|X_{i} v\right\|_{\alpha-\delta, N}\|v\|_{\alpha+\delta, N}$. This concludes the proof of Lemma 3.5. 
We now write the term containing $\tilde{K} A$ as

$$
\langle B u, \tilde{K} A u\rangle_{\varepsilon^{\prime}-1}=\sum_{i=1}^{m}\left(\left\langle X_{i} B u, X_{i} A u\right\rangle_{\varepsilon^{\prime}-1}+R_{i}\right),
$$

and we apply Lemma 3.2(e) with $f=B u, g=X_{i} A u, X=X_{i}^{T}$. Then we find

$$
\left|R_{i}\right| \leq\|B u\|_{-1, N}\left\|X_{i} A u\right\|_{2 \varepsilon^{\prime}-1} \leq\|B u\|_{-1, N}^{2}+\left\|X_{i} A u\right\|_{2 \varepsilon^{\prime}-1}^{2} .
$$

By Lemma 3.2(b), the first term is bounded by $B^{2}$. Using Lemma 3.2(c) to polarize the scalar product in (3.16) we thus get

$$
\left|\langle B u, \tilde{K} A u\rangle_{\varepsilon^{\prime}-1}\right| \leq \mathrm{B}^{2}+C \sum_{i=1}^{m}\left\|X_{i} B u\right\|_{-1}^{2}+C \sum_{i=1}^{m}\left\|X_{i} A u\right\|_{2 \varepsilon^{\prime}-1}^{2} .
$$

The term involving $\left\|X_{i} B u\right\|_{-1}^{2}$ is bounded by $\mathrm{B}^{2}$ as in (3.13). The last term is bounded by Lemma 3.5, yielding

$$
\begin{aligned}
\left|\langle B u, \tilde{K} A u\rangle_{\varepsilon^{\prime}-1}\right| \leq & \mathrm{B}^{2}+C\left|\left\langle K_{y} A u, A u\right\rangle_{2 \varepsilon^{\prime}-1}\right|+C \sum_{i=1}^{m}\left\|X_{i} A u\right\|_{-1, \tilde{N}}^{2} \\
& +C\|A u\|_{4 \varepsilon^{\prime}-1, \tilde{N}}^{2} .
\end{aligned}
$$

The last term in this expression is bounded by $\mathrm{B}^{2}$ by the induction hypothesis if we choose $\varepsilon^{\prime} \leq \varepsilon / 4$. The term containing $X_{i} A u$ can be bounded by $\mathrm{B}^{2}$ as in (3.13), so the only term that remains to be bounded is $\left|\left\langle K_{y} A u, A u\right\rangle_{2 \varepsilon^{\prime}-1}\right|$. By polarizing the estimate obtained by Lemma 3.2(c), one gets

$$
\left|\left\langle K_{y} A u, A u\right\rangle_{2 \varepsilon^{\prime}-1}\right| \leq C\|A u\|_{4 \varepsilon^{\prime}-1}^{2}+C\left\|K_{y} A u\right\|_{-1}^{2} .
$$

The first term is bounded by $\mathrm{B}^{2}$ using the induction assumption. The second term is bounded by $\mathrm{B}^{2}$ exactly like (3.14) above. Summing all these bounds this proves (3.7) and hence the inductive step is completed.

Since $K$ was assumed to satisfy $\mathcal{K}_{1}$ (or $\mathcal{K}_{0}$ ), we see that after $M$ inductive steps the proof of Theorem 3.1 is complete.

\section{Global Estimate}

The results of the previous section were restricted to functions $u$ with well-localized compact support. In this section, we are interested in getting bounds for every $u \in \mathrm{S}_{n}$. The main estimate of this section is given by

Theorem 4.1 Assume $K$ is in $\mathcal{K}_{1}$ or in $\mathcal{K}_{0}$ and let $K_{y}=K+$ iy be as above. For every $\varepsilon>0$, there exist constants $\delta>0$ and $C>0$ such that for the norms defined by (3.2) one has

$$
\|u\|_{\delta, \delta} \leq C\left(\|u\|_{0, \varepsilon}+\left\|K_{y} u\right\|\right)
$$

holds for every $u \in \mathrm{S}_{n}$. The constants $C$ and $\delta$ are independent of $y$ if $K \in \mathcal{K}_{1}$. 
Since $S^{\delta, \delta}$ is compactly embedded into $\mathrm{L}^{2}$, this result implies:

Corollary 4.2 Let $K$ be as above. If there exist constants $\varepsilon, C>0$ such that

$$
\|u\|_{0, \varepsilon} \leq C(\|u\|+\|K u\|),
$$

then $K$ has compact resolvent when considered as an operator acting on $\mathrm{L}^{2}$.

Proof of the Corollary. Combining (4.1) with (4.2), we get

$$
\|u\|_{\delta, \delta} \leq C(\|u\|+\|K u\|) .
$$

This implies that for $\lambda$ outside of the spectrum of $K$, the operator $(K-\lambda)^{-1}$ is bounded from $\mathrm{L}^{2}$ into $S^{\delta, \delta}$. By Lemma 3.2(a), it is therefore compact.

Proof of Theorem 4.1. Let $\varepsilon_{*}$ and $N_{*}$ be the values of the constants obtained in estimate (3.1) of Theorem 3.1. Observe that Theorem 3.1 also holds for any bigger value of $N_{*}$, and we will assume $N_{*}$ is sufficiently large.

We choose $\varepsilon>0$. As a first step, we will show that there exist constants $\delta$ and $C$ such that, for any $x \in \mathbf{R}^{n}$ and $u \in \mathcal{C}_{0}^{\infty}(\mathcal{B}(x))$, the following estimate holds:

$$
\|u\|_{\delta, \delta} \leq C\left(1+\|x\|^{2}\right)^{-N_{*}}\|u\|_{\varepsilon_{*}}+C\left(1+\|x\|^{2}\right)^{\varepsilon / 2}\|u\| .
$$

Denote by $J$ the smallest integer for which

$$
J \geq 1+\frac{8 N_{*}}{\varepsilon},
$$

and define

$$
\delta=\min \left\{2 N_{*}, \frac{\varepsilon}{2}, \frac{\varepsilon_{*}}{J}\right\} .
$$

First, we note that when $A$ is a positive self-adjoint operator on some Hilbert space $\mathcal{H}$, one has the estimate

$$
\|A u\|^{J} \leq C\left\|A^{J} u\right\|\|u\|^{J-1},
$$

whenever both expressions make sense. In the case $J=2^{j}$ for $j$ an integer, this can be seen by a repeated application of the Cauchy-Schwarz inequality. It was shown in [KS59] to hold in the general case as well.

We next use Jensen's inequality to write

$$
\left(1+\|x\|^{2}\right)^{N_{*}+\delta / 2}\left\|\Lambda^{\delta} u\right\| \leq C\left(\frac{\left\|\Lambda^{\delta} u\right\|}{\|u\|}\right)^{J}\|u\|+C\left(1+\|x\|^{2}\right)^{\left(N_{*}+\delta / 2\right)\left(1+\frac{1}{J-1}\right)}\|u\| .
$$

Dividing this expression by $\left(1+\|x\|^{2}\right)^{N_{*}}$ and using the definition of $J$, we get

$$
\begin{aligned}
\left(1+\|x\|^{2}\right)^{\delta / 2}\left\|\Lambda^{\delta} u\right\| \leq & C\left(1+\|x\|^{2}\right)^{-N_{*}}\left(\frac{\left\|\Lambda^{\delta} u\right\|}{\|u\|}\right)^{J}\|u\| \\
& +C\left(1+\|x\|^{2}\right)^{\left(N_{*}+\delta / 2\right)\left(1+\varepsilon /\left(8 N_{*}\right)\right)-N_{*}}\|u\| .
\end{aligned}
$$


Using (4.5), the fact that $\frac{\varepsilon}{8 N_{*}} \leq \frac{\varepsilon-\delta}{2 N_{*}+\delta}$ by (4.4), and $u \in \mathcal{C}_{0}^{\infty}(\mathcal{B}(x))$, we get (4.3).

In order to prove Theorem 4.1, we use the following partition of unity. Let $\chi_{0}$ : $\mathbf{R} \rightarrow[0,1]$ be a $\mathcal{C}^{\infty}$ function with support in $|x|<1$ and satisfying $\sum_{i \in \mathbf{Z}} \chi_{0}(x-i)=1$ for all $x \in \mathbf{R}$. The family of functions

$$
\mathcal{P}=\left\{\chi_{x}: \mathbf{R}^{n} \rightarrow[0,1] \mid x \in \mathbf{Z}^{n}\right\},
$$

defined by

$$
\chi_{x}(z)=\prod_{j=1}^{n} \chi_{0}\left(z_{j}-x_{j}\right),
$$

is therefore a partition of unity for $\mathbf{R}^{n}$. By construction, when $x, x^{\prime} \in \mathbf{Z}$ then $\chi_{x}$ and $\chi_{x^{\prime}}$ have disjoint support if there exists at least one index $j$ with $\left|x_{j}-x_{j}^{\prime}\right| \geq 2$. We can therefore split $\mathcal{P}$ into subsets $\left.\mathcal{P}_{k}\right|_{k=1, \ldots, 3^{n}}$ such that any two different functions belonging to the same $\mathcal{P}_{k}$ have disjoint supports.

Consider next an arbitrary function $u \in S_{n}$. We define $u_{x}=\chi_{x} u$, and then the construction of the $\mathcal{P}_{k}$ implies

$$
\sum_{x \in \mathbf{Z}^{n}}\left\|u_{x}\right\|_{0, \varepsilon} \leq 3^{n}\|u\|_{0, \varepsilon} .
$$

Using (4.3), then Theorem 3.1 and (4.6), we find

$$
\begin{aligned}
\|u\|_{\delta, \delta} & \leq \sum_{x \in \mathbf{Z}^{n}}\left\|u_{x}\right\|_{\delta, \delta} \leq C \sum_{x \in \mathbf{Z}^{n}}\left(\left(1+\|x\|^{2}\right)^{-N_{*}}\left\|u_{x}\right\|_{\varepsilon_{*}}+\left(1+\|x\|^{2}\right)^{\varepsilon / 2}\left\|u_{x}\right\|\right) \\
& \leq C \sum_{x \in \mathbf{Z}^{n}}\left(\left\|u_{x}\right\|+\left(1+\|x\|^{2}\right)^{-N_{*}}\left\|K_{y} u_{x}\right\|+\left(1+\|x\|^{2}\right)^{\varepsilon / 2}\left\|u_{x}\right\|\right) \\
& \leq C 3^{n}\left(\|u\|+\|u\|_{0, \varepsilon}\right)+C \sum_{x \in \mathbf{Z}^{n}}\left(1+\|x\|^{2}\right)^{-N_{*}}\left\|K_{y} u_{x}\right\| .
\end{aligned}
$$

For $k \in\left\{1, \ldots, 3^{n}\right\}$ we now define

$$
f_{k}=\sum_{\chi_{k, \ell} \in \mathcal{P}_{k}}\left(1+\|x\|^{2}\right)^{-N_{*}} \chi_{k, \ell} .
$$

With this notation, we have

$$
\|u\|_{\delta, \delta} \leq C\|u\|_{0, \varepsilon}+C \sum_{k=1}^{3^{n}}\left\|K_{y} f_{k} u\right\| .
$$

The claim (4.1) thus follows if we can show that

$$
\left\|K_{y} f_{k} u\right\| \leq C\|u\|+C\left\|K_{y} u\right\| .
$$

Since the $f_{k}$ are bounded functions, it suffices to estimate $\left\|\left[K, f_{k}\right] u\right\|$. By construction, every derivative of $f_{k}$ decays like $\left(1+\|x\|^{2}\right)^{-N_{*}}$.

Note that for sufficiently large $N_{*}$, the functions $\left[X_{j}, f_{k}\right]$ and $\left[X_{k},\left[X_{j}, f_{k}\right]\right]$ are bounded. Since Theorem 3.1 allows us to choose $N_{*}$ as large as we wish, (4.7) follows from the estimate $\left\|X_{i} u\right\|^{2} \leq\|u\|\left\|K_{y} u\right\|$. 


\subsection{Cusp}

Our statement about the cusp-like shape of the spectrum of $K$ is now a consequence of Theorem 4.1.

Theorem 4.3 Let $K \in \mathbf{P o l}_{2}^{N}$ be of the type (1.1). Assume that the closure of $K$ in $\mathrm{L}^{2}$ is $m$-accretive and that $K \in \mathcal{K}_{1}$. Assume furthermore that there exist constants $\varepsilon, C>0$ such that

$$
\|u\|_{0, \varepsilon} \leq C\left(\|u\|+\left\|K_{y} u\right\|\right),
$$

for all $y \in \mathbf{R}$. Then, the spectrum of $K$ (as an operator on $\mathrm{L}^{2}$ ) is contained in the cusp

$$
\left\{\lambda \in \mathbf{C}|\operatorname{Re} \lambda \geq 0,| \operatorname{Im} \lambda \mid \leq C(1+\operatorname{Re} \lambda)^{\nu}\right\},
$$

for some positive constants $C$ and $\nu$.

Remark 4.4 In principle, our proofs give a constructive upper bound on $\nu$. However, no attempt has been made to optimize this bound.

Proof. The proof follows very closely that of Theorem 4.1 in [HN02], however we give the details for completeness. One ingredient we need is the following lemma:

Lemma 4.5 Let $A: \mathrm{L}^{2} \rightarrow \mathrm{L}^{2}$ be a maximal accretive operator that has $\mathrm{S}_{n}$ as a core. Assume there exist constants $C, \alpha>0$ for which

$$
\|A u\| \leq C\|u\|_{\alpha, \alpha}, \quad \forall u \in \mathrm{S}_{n} .
$$

Then, for every $N \in \mathbf{N}$, there exists a constant $C_{N}$ such that

$$
\left\|A^{1 / N} u\right\| \leq C_{N}\|u\|_{\alpha / N, \alpha / N}, \quad \forall u \in S^{\alpha / N, \alpha / N} .
$$

Proof. By Lemma 3.2(b), one can bound $\|u\|_{\alpha, \alpha}$ by

$$
\|u\|_{\alpha, \alpha} \leq C\left\|\left(\Lambda^{\alpha / 2 N} \bar{\Lambda}^{\alpha / N} \Lambda^{\alpha / 2 N}\right)^{N} u\right\|
$$

The generalized Heinz inequality presented in [Kat61] then yields

$$
\left\|A^{1 / N} u\right\| \leq C_{N}\left\|\Lambda^{\alpha / 2 N} \bar{\Lambda}^{\alpha / N} \Lambda^{\alpha / 2 N} u\right\|
$$

This concludes the proof of Lemma 4.5.

We now turn to the proof of Theorem 4.3. Since $K \in \mathbf{P o l}_{2}^{N}$, one has for $\alpha=$ $\max \{2, N\}$ the bound

$$
\|(K+1) u\| \leq C\|u\|_{\alpha, \alpha}, \quad \forall u \in \mathrm{S}_{n} .
$$

By Lemma 4.5, one can find for every $\delta>0$ an integer $M>0$ and a constant $C$ such that:

$$
\left\langle u,\left((K+1)^{*}(K+1)\right)^{1 / M} u\right\rangle \leq C\|u\|_{\delta, \delta}^{2},
$$


Furthermore, Theorem 4.1 together with (4.8) yields constants $C$ and $\delta$ such that for every $u \in \mathrm{S}_{n}$ and every $y \in \mathbf{R}$ :

$$
\|u\|_{\delta, \delta}^{2} \leq C\left(\|u\|^{2}+\|(K+i y) u\|^{2}\right) .
$$

Since $K$ is $m$-accretive by assumption, we can apply [HN02, Prop. B.1] to get the estimate

$$
\begin{aligned}
\frac{1}{4}|z+1|^{2 / M}\|u\|^{2} & \leq\left\langle\left((K+1)^{*}(K+1)\right)^{1 / M} u, u\right\rangle+\|(K-z) u\|^{2} \\
& \leq C\|u\|_{\delta, \delta}^{2}+\|(K-z) u\|^{2},
\end{aligned}
$$

where the second line is a consequence of (4.9). Using (4.10) and the triangle inequality for $z=\operatorname{Re} z+i \operatorname{Im} z$, we get

$$
\frac{1}{4}|z+1|^{\varepsilon / M}\|u\|^{2} \leq C\left((1+\operatorname{Re} z)^{2}\|u\|^{2}+\|(K-z) u\|^{2}\right) .
$$

Together with the compactness of the resolvent of $K$, this immediately implies that every $\lambda$ in the spectrum of $K$ satisfies the inequality

$$
\frac{1}{4}|\lambda+1|^{\varepsilon / M}\|u\|^{2} \leq C(1+\operatorname{Re} \lambda)^{2}\|u\|^{2} .
$$

This concludes the proof of Theorem 4.3.

\section{Examples}

We present two examples in this section: A first, very simple one, and a second which was the main motivation for this paper.

\subsection{Langevin equation for a simple anharmonic oscillator}

Our first example consists of one anharmonic oscillator which is in contact with a stochastic heat bath at temperature $T$. The Hamiltonian of the oscillator is given by

$$
H(p, q)=\frac{p^{2}}{2}+\frac{\nu^{2} q^{2}}{2}+\varepsilon \frac{q^{4}}{4} .
$$

For this model the associated spectral problem can be solved explicitly when $\varepsilon=0$, because it is an harmonic oscillator. The spectrum lies in a cone as shown in Fig. 2. We also show that in first order perturbation theory in $\varepsilon$, the spectrum seems to form a non-trivial cusp, but this result remains conjectural, because of non-uniformity of our bounds.

The Langevin equation for this system is

$$
d p=-\nu^{2} q d t-\varepsilon q^{3} d t-\gamma p d t+\sqrt{2 \gamma T} d w(t), \quad d q=p d t,
$$

where $\gamma>0$ measures the strength of the interaction between the oscillator and the bath. Denote by $(\Omega, \mathbf{P})$ the probability space on which the Wiener process $w(t)$ is defined. We write $\varphi_{t, \omega}(x)$ with $\omega \in \Omega$ for the solution at time $t$ for (5.1) with initial 
condition $x=(p, q)$ and realization $\omega$ of the white noise. The corresponding semigroups acting on observables and on measures on $\mathbf{R}^{2}$ are given by

$$
\begin{aligned}
\left(T_{t} f\right)(x) & =\int_{\Omega}\left(f \circ \varphi_{t, \omega}(x)\right) d \mathbf{P}(\omega), \\
\left(T_{t}^{*} \mu\right)(A) & =\int_{\Omega}\left(\mu \circ \varphi_{t, \omega}^{-1}(A)\right) d \mathbf{P}(\omega),
\end{aligned}
$$

where $A \subset \mathbf{R}^{2}$ is a Borel set. It is well-known that

$$
d \mu_{T}(p, q)=\exp (-H(p, q) / T) d p d q
$$

is the only stationary solution for $(5.2 \mathrm{~b})$.

The Itô formula yields for $f_{t}=T_{t} f$ the Fokker-Planck equation given by

$$
\partial_{t} f_{t}=\gamma T \partial_{p}^{2} f_{t}+p \partial_{q} f_{t}-\left(\nu^{2} q+\varepsilon q^{3}+\gamma p\right) \partial_{p} f_{t} .
$$

We study (5.3) in the space $\mathcal{H}_{\beta}=\mathrm{L}^{2}\left(\mathbf{R}^{2}, d \mu_{T}\right)$. and make the change of variables $f_{t}=\exp (H /(2 T)) F_{t}$ in order to work in the unweighted space $\mathcal{H}_{0}=\mathrm{L}^{2}\left(\mathbf{R}^{2}, d p d q\right)$. Equation (5.3) then becomes $\partial_{t} F_{t}=-\tilde{\mathcal{L}}_{\varepsilon} F_{t}$, where the differential operator $\tilde{\mathcal{L}}_{\varepsilon}$ is given by

$$
\tilde{\mathcal{L}}_{\varepsilon}=-\gamma T \partial_{p}^{2}+\frac{\gamma}{4 T} p^{2}-\frac{\gamma}{2}-p \partial_{q}+\nu^{2} q \partial_{p}+\varepsilon q^{3} \partial_{p} .
$$

By rescaling time, $p$ and $q$, one can bring $\tilde{\mathcal{L}}_{\varepsilon}$ to the form

$$
\mathcal{L}_{\varepsilon}=\frac{1}{2}\left(-\partial_{p}^{2}+p^{2}-1\right)+\alpha\left(q \partial_{p}-p \partial_{q}\right)+c \varepsilon q^{3} \partial_{p},
$$

where $\alpha=2 \sqrt{2 T} \nu / \gamma$ and $c>0$.

The operator $K=\mathcal{L}_{\varepsilon}$ is thus of the type (1.1) with $X_{0}=\alpha\left(q \partial_{p}-p \partial_{q}\right)+c \varepsilon q^{3} \partial_{p}$ and $X_{1}=\partial_{p}$. We now verify the conditions of Section 2.2. It is obvious that these vector fields are of polynomial growth, thus condition $a$ is satisfied. Since $\left[X_{1}, X_{0}\right]=-\alpha \partial_{q}$, the operator $\mathcal{L}_{\varepsilon}$ satisfies condition $b_{1}$ as well, and so the conclusion of Theorem 4.1 holds. Proceeding like in [EH00, Prop. 3.7], one shows an estimate of the type (4.8) (see also the proof of Theorem 5.5 below, where details are given). Therefore, Theorem 4.3 applies, showing that the spectrum of $\mathcal{L}_{\varepsilon}$ is located in a cusp-shaped region. In fact, we show in the next subsection that the cusp is a cone when $\varepsilon=0$, and then we study its perturbation to first order in $\varepsilon$.

\subsubsection{First-order approximation of the spectrum of $\mathcal{L}_{\varepsilon}$}

We will explicitly compute the spectrum and the corresponding eigenfunctions for $\mathcal{L}_{0}$ and then (formally) apply first-order perturbation theory to get an approximation to the spectrum of $\mathcal{L}_{\varepsilon}$. We introduce the "creation and annihilation" operators

$$
a=\frac{p+\partial_{p}}{\sqrt{2}}, \quad a^{*}=\frac{p-\partial_{p}}{\sqrt{2}}, \quad b=\frac{q+\partial_{q}}{\sqrt{2}}, \quad b^{*}=\frac{q-\partial_{q}}{\sqrt{2}},
$$


in terms of which $\mathcal{L}_{\varepsilon}$ can be written as

$$
\mathcal{L}_{\varepsilon}=a^{*} a+\alpha\left(b^{*} a-a^{*} b\right)+c \varepsilon q^{3} \partial_{p} .
$$

With this notation, it is fairly easy to construct the spectrum of $\mathcal{L}_{0}$. Note first that 0 is an eigenvalue for $\mathcal{L}$ with eigenfunction $\exp \left(-p^{2} / 2-q^{2} / 2\right)$. This is actually the vacuum state for the two-dimensional harmonic oscillator in quantum mechanics (which is given by $a^{*} a+b^{*} b$ ), so we call this eigenfunction $|\Omega\rangle$.

A straightforward calculation shows that the creation operators $c_{ \pm}^{*}$ defined by

$$
c_{ \pm}^{*}=a^{*}+\beta_{ \pm} b^{*}, \quad \beta_{ \pm}=-\frac{1}{2 \alpha} \pm i \frac{\sqrt{4 \alpha^{2}-1}}{2 \alpha}
$$

satisfy the following commutation relation with $\mathcal{L}_{0}$ :

$$
\left[\mathcal{L}_{0}, c_{ \pm}^{*}\right]=\lambda_{ \pm} c_{ \pm}^{*}, \quad \lambda_{ \pm}=\frac{1}{2} \pm i \frac{\sqrt{4 \alpha^{2}-1}}{2}=-\frac{\alpha}{\beta_{ \pm}} .
$$

Therefore, $\lambda_{0}^{n, m}=n \lambda_{+}+m \lambda_{-}$with $n$ and $m$ positive integers are eigenvalues for $\mathcal{L}_{0}$ with eigenvectors given by

$$
\left(c_{+}^{*}\right)^{n}\left(c_{-}^{*}\right)^{m}|\Omega\rangle \text {. }
$$

We conclude that for $\alpha>1 / 2$ the spectrum of $\mathcal{L}_{0}$ consists of a triangular grid located inside a cone (see Figure 2).

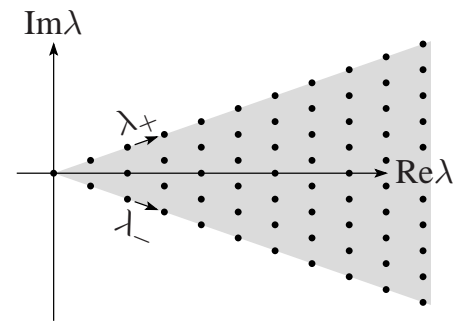

Figure 2 Spectrum of $\mathcal{L}_{0}$.

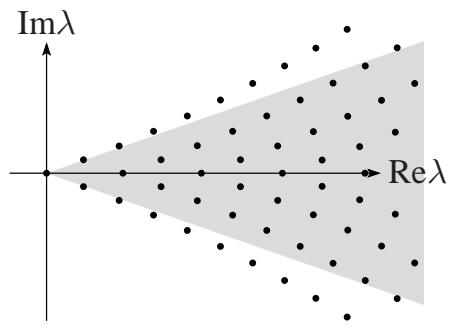

Figure 3 Approximate spectrum of $\mathcal{L}_{\varepsilon}$.

Remark 5.1 Although the spectrum of $\mathcal{L}_{0}$ is located inside a sector, $\mathcal{L}_{0}$ is not sectorial since the closure of its numerical range is the half-plane $\operatorname{Re} \lambda \geq 0$.

In order to do first-order perturbation theory for the spectrum of $\mathcal{L}_{\varepsilon}$ we also need the eigenvectors for $\mathcal{L}_{0}^{*}$, which can be obtained by applying successively $d_{+}^{*}$ and $d_{-}^{*}$ to $|\Omega\rangle$, where

$$
d_{ \pm}^{*}=a^{*}-\beta_{\mp} b^{*} .
$$

With this notation, $\left(d_{+}^{*}\right)^{n}\left(d_{-}^{*}\right)^{m}|\Omega\rangle$ is an eigenvector of $\mathcal{L}_{0}^{*}$ with eigenvalue $\bar{\lambda}_{0}^{n, m}$. By first-order perturbation theory, the eigenvalues of $\mathcal{L}_{\varepsilon}$ are approximated by

$$
\lambda_{\varepsilon}^{n, m} \approx \lambda_{0}^{n, m}+c \varepsilon \delta_{n, m}, \quad \delta_{n, m}=\frac{\left\langle\Omega\left|d_{-}^{m} d_{+}^{n} q^{3} \partial_{p}\left(c_{+}^{*}\right)^{n}\left(c_{-}^{*}\right)^{m}\right| \Omega\right\rangle}{\left\langle\Omega\left|d_{-}^{m} d_{+}^{n}\left(c_{+}^{*}\right)^{n}\left(c_{-}^{*}\right)^{m}\right| \Omega\right\rangle} .
$$


The resulting spectrum ${ }^{2}$ is shown in Figure 3 (the sector containing the spectrum of $\mathcal{L}_{0}$ is shown in light gray for comparison). One clearly sees that the boundary of the sector bends to a cusp. A (lengthy) explicit computation also shows that

$$
\delta_{n, 0}=-12 n(n-1) \frac{\bar{\lambda}_{+}}{\sqrt{4 \alpha^{2}-1}}+9 n \frac{i \alpha}{\sqrt{4 \alpha^{2}-1}} .
$$

In principle this confirms the cusp-like shape of the boundary, were it not for the nonuniformity of the perturbation theory (in $n$ ).

\subsection{A model of heat conduction}

In this subsection, we apply our results to the physically more interesting case of a chain of nearest-neighbor interacting anharmonic oscillators coupled to two heat baths at different temperatures. We model the chain by the deterministic Hamiltonian system given by

$$
H=\sum_{i=0}^{N}\left(\frac{p_{i}^{2}}{2}+V_{1}\left(q_{i}\right)\right)+\sum_{i=1}^{N} V_{2}\left(q_{i}-q_{i-1}\right) .
$$

(We will give conditions on the potentials $V_{1}$ and $V_{2}$ later on.) In order too keep notations short, we assume $p_{i}, q_{i} \in \mathbf{R}$, but one could also take them in $\mathbf{R}^{d}$ instead. The two heat baths are modeled by classical free field theories $\varphi_{L}$ and $\varphi_{R}$ with initial conditions drawn randomly according to Gibbs measures at respective inverse temperatures $\beta_{L}$ and $\beta_{R}$. (We refer to [EPR99a] for a more detailed description of the model.) It is shown in [EPR99a] that this model is equivalent to the following system of stochastic differential equations:

$$
\begin{array}{rlr}
d q_{i} & =p_{i} d t, & i=0, \ldots, N, \\
d p_{0} & =-V_{1}^{\prime}\left(q_{0}\right) d t+V_{2}^{\prime}\left(\tilde{q}_{1}\right) d t+r_{L} d t, & \\
d p_{j} & =-V_{1}^{\prime}\left(q_{j}\right) d t-V_{2}^{\prime}\left(\tilde{q}_{j}\right) d t+V_{2}^{\prime}\left(\tilde{q}_{j+1}\right) d t, & j=1, \ldots, N-1, \\
d p_{N} & =-V_{1}^{\prime}\left(q_{N}\right) d t-V_{2}^{\prime}\left(\tilde{q}_{N}\right) d t+r_{R} d t, & \\
d r_{L} & =-\gamma_{L} r_{L} d t+\lambda_{L}^{2} \gamma_{L} q_{0} d t-\lambda_{L} \sqrt{2 \gamma_{L} T_{L}} d w_{L}(t), & \\
d r_{R} & =-\gamma_{R} r_{R} d t+\lambda_{R}^{2} \gamma_{R} q_{N} d t-\lambda_{R} \sqrt{2 \gamma_{R} T_{R}} d w_{R}(t),
\end{array}
$$

where $T_{i}=\beta_{i}^{-1}, \gamma_{i}$ are positive constants describing the coupling of the chain to the heat baths, and $w_{i}$ are two independent Wiener processes. The variables $r_{L}$ and $r_{R}$ describe the internal state of the heat baths. If $T_{L}=T_{R}=T$, the equilibrium measure for this system is $d \mu_{T}(p, q, r)=\exp (-G(p, q, r) / T) d p d q d r$, where the "energy" $G$ is given by the expression

$$
G(p, q, r)=H(p, q)+\frac{r_{L}^{2}}{2 \lambda_{L}^{2}}-q_{0} r_{L}+\frac{r_{R}^{2}}{2 \lambda_{R}^{2}}-q_{N} r_{R} .
$$

\footnotetext{
${ }^{2}$ Actually the set $\left\{\lambda_{0}^{n, m}+c \varepsilon \delta_{n, m} \mid n, m \geq 0\right\}$.
} 
If $T_{L} \neq T_{R}$, there is no way of guessing the invariant measure for the system. We can nevertheless make the construction of Section 5.1 with the reference measure $d \mu_{\tilde{T}}$ for some temperature

$$
\tilde{T}>\max \left\{T_{L}, T_{R}\right\}
$$

which is a stability condition, as one can see in (5.6) below. The resulting operator $K=\mathcal{L}$ is given by

$$
\mathcal{L}=X_{L}^{*} X_{L}+X_{R}^{*} X_{R}+f_{L}^{2}+f_{R}^{2}+X_{0}
$$

where

$$
\begin{aligned}
X_{L, R}= & \lambda_{L, R} \sqrt{\gamma_{L, R} T_{L, R}} \partial_{r_{L, R}} \\
f_{L, R}= & \sqrt{\gamma_{L, R}\left(T_{L, R} / \tilde{T}-1\right)}\left(r_{L, R}-\lambda_{L, R} q_{0, N}\right) \\
X_{0}= & \nabla_{q} H \nabla_{p}-\nabla_{p} H \nabla_{q}+b_{L}\left(r_{L}-\lambda_{L}^{2} q_{0}\right) \partial_{r_{L}}-r_{L} \partial_{p_{0}} \\
& +b_{R}\left(r_{L}-\lambda_{R}^{2} q_{N}\right) \partial_{r_{R}}-r_{L} \partial_{p_{N}},
\end{aligned}
$$

with

$$
b_{L, R}=\frac{\gamma_{L, R}}{\lambda_{L, R}^{2} \tilde{T}^{2}}\left(T_{L, R}-\tilde{T}\right) .
$$

We are now in a position to express the conditions of Section 2.2 in terms of sufficient conditions on the potentials of the model. The first two assumptions guarantee that $\mathcal{L}$ is in $\mathcal{K}_{1}$.

Assumption 1 There exist real numbers $n, m>0$ such that $D^{\alpha} V_{1} \in \mathbf{P o l}_{0}^{2 n-\alpha}$ and $D^{\alpha} V_{2} \in \mathbf{P o l}_{0}^{2 m-\alpha}$ for $\alpha \leq 2$.

Assumption 2 There exists a constant $c>0$ such that $V_{2}^{\prime \prime}(x)>c$ for all $x \in \mathbf{R}$.

Remark 5.2 The second assumption states that there is a non-vanishing coupling between neighboring particles in every possible state of the chain.

The verification that these assumptions imply $a$ is easy, and the verification that $b_{1}$ holds can be found in [EPR99a, EH00].

Proposition 5.3 Let $\mathcal{L}$ be defined as above and let $V_{1}$ and $V_{2}$ fulfill Assumptions 1 and 2 above. Then $\mathcal{L}$ satisfies the assumptions of Theorem 4.1 and satisfies Eq.(4.1) with $C$ and $\delta$ independent of $y$.

In order to show that the spectrum of $\mathcal{L}$ is located in a cusp-shaped region (i.e. that the hypotheses of Theorem 4.3 hold), two more assumptions have to be made on the asymptotic behaviour of $V_{1}$ and $V_{2}$ :

Assumption 3 The exponents $n$ and $m$ appearing in Assumption 1 satisfy $1<n<m$. 
Remark 5.4 The physical interpretation of the condition $n<m$ (actually $1 \leq n \leq m$ would probably work as well, see [RBT02b], but we could not apply directly the results of [EH00]) goes as follows. If $n>m$, the relative strength of the coupling between neighboring particles decreases as the energy of the chain tends to infinity. Therefore, an initial condition where all the energy of the chain is concentrated into one single oscillator is "metastable" in the sense that the energy gets transmitted only very slowly to the neighboring particles and eventually to the heat baths. As a consequence, it is likely that the convergence to a stationary state is no longer exponential in this case, and so the operator $\mathcal{L}$ has probably not a compact resolvent anymore.

Our last assumption states that the potentials and the resulting forces really grow asymptotically like $|x|^{n}$ and $|x|^{m}$ respectively (and not just "slower than").

Assumption 4 The potentials $V_{1}$ and $V_{2}$ satisfy the conditions

$$
\begin{aligned}
& V_{1}(x) \geq c_{1}\left(1+\|x\|^{2}\right)^{n}-c_{2}, \quad x V_{1}^{\prime}(x) \geq c_{3}\left(1+\|x\|^{2}\right)^{n}-c_{4}, \\
& V_{2}(x) \geq c_{5}\left(1+\|x\|^{2}\right)^{m}-c_{6}, \quad x V_{2}^{\prime}(x) \geq c_{7}\left(1+\|x\|^{2}\right)^{m}-c_{8},
\end{aligned}
$$

for all $x \in \mathbf{R}$ and for some positive constants $c_{i}$.

Theorem 5.5 Let $\mathcal{L}$ be defined as above and let $V_{1}$ and $V_{2}$ fulfill assumptions 1-4 above. Then, $\mathcal{L}$ has compact resolvent and there exist positive constants $C$ and $N$ such that the spectrum of $\mathcal{L}$ is contained in the cusp

$$
\left\{\lambda \in \mathbf{C} \mid \operatorname{Re} \lambda \geq 0 \quad \text { and } \operatorname{Im} \lambda \leq C(1+|\operatorname{Re} \lambda|)^{N}\right\} .
$$

Proof. We will apply Theorem 4.3, and need to check its assumptions. It has been shown in [EH00, Prop. B.3] that $\mathcal{L}$ is $m$-accretive. The fact that $\mathcal{L} \in \mathcal{K}_{1}$ was checked above, and (4.8) was shown for $y=0$ in [EH00, Prop. 3.7]. However, closer inspection of that proof reveals that whenever $X_{0}$ was used, it only appeared inside a commutator. Therefore, we can replace it by $X_{0}+i y$ without changing the bounds. Thus, we have checked all the assumptions of Theorem 4.3 and the proof of Theorem 5.5 is complete.

\section{A Proof of Lemma 3.2}

The points $a$ and $b$ of Lemma 3.2 are standard results in the theory of pseudodifferential operators (see e.g. [Hör85, Vol. III] or, more specifically, [BC94, HT94a, HT94b]). The point $c$ is an immediate consequence of the Cauchy-Schwarz inequality combined with $a$. In order to prove the points $d$ and $e$, we first show the following intermediate result:

Lemma A.1 Let $f: \mathbf{R}^{n} \rightarrow \mathbf{R}$ and $\alpha \in \mathbf{R}$. Let $k$ be the smallest even integer such that $|\alpha| \leq k$. Then, if $f$ satisfies

$$
\sup _{y \in \mathbf{R}^{n}}\left|\partial^{\delta} f(y)\right|<\kappa, \quad \forall|\delta| \leq k,
$$

the corresponding operator of multiplication is bounded from $S^{\alpha, \beta}$ into $S^{\alpha, \beta}$ and its operator norm is bounded by $C \kappa$. The constant $C$ depends only on $\alpha$ and $\beta$. 
Proof. By the definition of $S^{\alpha, \beta}$, it suffices to show that the operator $\Lambda^{\alpha} f \Lambda^{-\alpha}$ is bounded by $C \kappa$ from $\mathrm{L}^{2}$ into $\mathrm{L}^{2}$. Since $f$ is obviously bounded by $\kappa$ as a multiplication operator from $\mathrm{L}^{2}$ into $\mathrm{L}^{2}$, it actually suffices to bound $\Lambda^{\alpha}\left[f, \Lambda^{-\alpha}\right]$. Assume first that $\alpha \in(0,2)$. In that case, we write

$$
\Lambda^{\alpha}\left[f, \Lambda^{-\alpha}\right]=C_{\alpha} \int_{0}^{\infty} z^{-\alpha / 2} \frac{\Lambda^{\alpha}}{z+\Lambda^{2}}\left[f, \Lambda^{2}\right] \frac{1}{z+\Lambda^{2}} d z .
$$

The commutator appearing in this expression can be written as

$$
\left[f, \Lambda^{2}\right]=\sum_{i=1}^{n}\left(2 \partial_{i} f \partial_{i}+\partial_{i}^{2} f\right)
$$

It is clear from basic Fourier analysis that $\left\|\partial_{i}\left(z+\Lambda^{2}\right)^{-1 / 2}\right\| \leq 1$ and therefore

$$
\left\|\left[f, \Lambda^{2}\right]\left(z+\Lambda^{2}\right)^{-1 / 2}\right\| \leq C \kappa .
$$

Furthermore, the spectral theorem tells us that for any function $F,\left\|F\left(\Lambda^{2}\right)\right\|$ is bounded by $\sup _{\lambda \geq 1} F(\lambda)$. Therefore there exists a constant $C$ independent of $z>0$ such that

$$
\left\|\Lambda^{\alpha}\left(z+\Lambda^{2}\right)^{-1}\right\| \leq \frac{C}{1+z^{1-\alpha / 2}} .
$$

Combining these estimates shows the claim when $\alpha \in(0,2)$. The case $\alpha=2$ follows from the boundedness of $\left[f, \Lambda^{2}\right] \Lambda^{-2}$. Values of $\alpha$ greater than 2 can be obtained by iterating the relation

$$
\Lambda^{\alpha+2} f \Lambda^{-\alpha-2}=\Lambda^{\alpha} f \Lambda^{-\alpha}+\Lambda^{\alpha}\left[f, \Lambda^{2}\right] \Lambda^{-\alpha-2} .
$$

Using (A.1), the fact that $\partial_{i}$ commutes with $\Lambda$, and the fact that $\partial_{i} \Lambda^{-2}$ is bounded, we can reduce this to the previous case, but with two more derivatives to control. The case $\alpha<0$ follows by considering adjoints. This concludes the proof of Lemma A.1.

Remark A.2 Since the direct and the inverse Fourier transforms both map $S^{\alpha, \beta}$ continuously into $S^{\beta, \alpha}$, the above lemma also holds for bounded functions of $\partial_{y}$ and not only for bounded functions of $y$.

We are now ready to turn to the

Proof of point $\boldsymbol{d}$. Let $X \in \mathbf{P o l}_{k}^{N}$. We first consider $\gamma \in(-2,0)$. Since, in Fourier space, $\Lambda^{2}$ is a multiplication operator by a real positive function, we can write

$$
\left[X, \Lambda^{\gamma}\right]=C_{\gamma} \int_{0}^{\infty} z^{\gamma / 2} \frac{1}{z+\Lambda^{2}}\left[X, \Lambda^{2}\right] \frac{d z}{z+\Lambda^{2}} .
$$

In order to bound this expression, we define $B=\left[X, \Lambda^{2}\right]$, commute $B$ with the resolvent, and obtain

$$
\left[X, \Lambda^{\gamma}\right]=C_{\gamma} \int_{0}^{\infty} z^{\gamma / 2} \frac{\Lambda^{2-\gamma} d z}{\left(z+\Lambda^{2}\right)^{2}} \Lambda^{\gamma-2} B+C_{\gamma} \int_{0}^{\infty} \frac{z^{\gamma / 2}}{\left(z+\Lambda^{2}\right)^{2}}\left[B, \Lambda^{2}\right] \frac{d z}{z+\Lambda^{2}}
$$


The first term equals $C_{\gamma}^{\prime} \Lambda^{\gamma-2} B$ because $\int_{0}^{\infty} z^{\gamma / 2} x^{2-\gamma}\left(z+x^{2}\right)^{-2} d z$ does not depend on $x>0$. This, in turn, is bounded from $S^{\alpha, \beta}$ into $S^{\alpha+1-k-\gamma, \beta-N}$ using $B \in \mathbf{P o l}_{k+1}^{N}$ and Lemma 3.2(b). To bound the second term, we rewrite

$$
\int_{0}^{\infty} \frac{z^{\gamma / 2}}{\left(z+\Lambda^{2}\right)^{2}}\left[B, \Lambda^{2}\right] \frac{d z}{z+\Lambda^{2}}=\int_{0}^{\infty} \frac{z^{\gamma / 2} \Lambda^{1-\gamma}}{\left(z+\Lambda^{2}\right)^{2}} \cdot \Lambda^{\gamma-1}\left[B, \Lambda^{2}\right] \Lambda^{-2} \cdot \frac{\Lambda^{2}}{z+\Lambda^{2}} d z .
$$

The factor $\Lambda^{2}\left(z+\Lambda^{2}\right)^{-1}$ is bounded from $S^{\alpha, \beta}$ into itself, uniformly in $z$. Using Lemma 3.2(b) as before, we see that the factor $\Lambda^{\gamma-1}\left[B, \Lambda^{2}\right] \Lambda^{-2}$ is bounded from $S^{\alpha, \beta}$ into $S^{\alpha+1-k-\gamma, \beta-N} \equiv S^{\alpha^{\prime}, \beta^{\prime}}$. Finally, using Lemma A.1 and counting powers, we see that the first factor has norm bounded by $\mathcal{O}\left(z^{-3 / 2}\right)$ for large $z$ and $\mathcal{O}\left(z^{\gamma / 2}\right)$ for $z$ near 0 as a map from $S^{\alpha^{\prime}, \beta^{\prime}}$ to itself. This proves the first statement of Lemma 3.2(d). The second one is proven similarly and is left to the reader.

Proof of point $\boldsymbol{e}$. Recall that we want to bound

$$
I=\left|\langle f, X g\rangle_{\alpha, \beta}-\left\langle X^{T} f, g\right\rangle_{\alpha, \beta}\right|,
$$

where $X \in \mathbf{P o l}_{k}^{N}$ and $X^{T}$ denotes the formal adjoint (in $\mathrm{L}^{2}$ ) of $X$. We write this as

$$
I=\left\langle\left[\bar{\Lambda}^{-2 \beta} \Lambda^{-2 \alpha}, X^{T}\right] \Lambda^{2 \alpha} \bar{\Lambda}^{2 \beta} f, g\right\rangle_{\alpha, \beta} .
$$

We rewrite the operator as

$$
\left[\bar{\Lambda}^{-2 \beta} \Lambda^{-2 \alpha}, X^{T}\right] \Lambda^{2 \alpha} \bar{\Lambda}^{2 \beta}=\bar{\Lambda}^{-2 \beta}\left[\Lambda^{-2 \alpha}, X^{T}\right] \Lambda^{2 \alpha} \bar{\Lambda}^{2 \beta}+\left[\bar{\Lambda}^{-2 \beta}, X^{T}\right] \bar{\Lambda}^{2 \beta}
$$

The second term is in $\mathbf{P o l}_{k-1}^{N}$ by inspection, and the required bound follows at once from Lemma 3.2(b,c). The first term is bounded similarly by using Lemma 3.2(d,b,c). This concludes the proof of Lemma 3.2.

\section{References}

[BC94] J.-M. BonY and J.-Y. Chemin. Espaces fonctionnels associés au calcul de WeylHörmander. Bull. Soc. Math. France 122, no. 1, (1994), 77-118.

[EH00] J.-P. ECKMANN and M. HAIRER. Non-equilibrium statistical mechanics of strongly anharmonic chains of oscillators. Comm. Math. Phys. 212, no. 1, (2000), 105-164.

[EPR99a] J.-P. ECKMAnn, C.-A. Pillet, and L. Rey-Bellet. Non-equilibrium statistical mechanics of anharmonic chains coupled to two heat baths at different temperatures. Comm. Math. Phys. 201, (1999), 657-697.

[EPR99b] J.-P. Eckmann, C.-A. Pillet, and L. Rey-Bellet. Entropy production in nonlinear, thermally driven hamiltonian systems. J. Stat. Phys. 95, (1999), 305-331.

[HN02] F. HÉRAU and F. NIER. Isotropic hypoellipticity and trend to equilibrium for the Fokker-Planck equation with high degree potential, 2002. Preprint.

[Hör85] L. Hörmander. The Analysis of Linear Partial Differential Operators I-IV. Springer, New York, 1985. 
[HT94a] D. HAROSKE and H. TRIEBEL. Entropy numbers in weighted function spaces and eigenvalue distributions of some degenerate pseudodifferential operators. I. Math. Nachr. 167, (1994), 131-156.

[HT94b] D. HAROSKE and H. TRIEBEL. Entropy numbers in weighted function spaces and eigenvalue distributions of some degenerate pseudodifferential operators. II. Math. Nachr. 168, (1994), 109-137.

[Kat61] T. Kato. A generalization of the Heinz inequality. Proc. Japan Acad. 37, (1961), 305-308.

[KS59] M. A. KRASNOSEL'SKII and P. E. SOBOLEVSKIĬ. Fractional powers of operators acting in Banach spaces. Dokl. Akad. Nauk SSSR 129, (1959), 499-502.

[RBT00] L. REY-Bellet and L. E. Thomas. Asymptotic behavior of thermal nonequilibrium steady states for a driven chain of anharmonic oscillators. Comm. Math. Phys. 215, no. 1, (2000), 1-24.

[RBT02a] L. REY-BeLlet and L. Thomas. Fluctuations of the entropy production in anharmonic chains, 2002. To be published in Ann. Henri Poincare.

[RBT02b] L. REY-BELLET and L. E. ThOMAS. Exponential convergence to non-equilibrium stationary states in classical statistical mechanics. Comm. Math. Phys. 225, no. 2, (2002), 305-329. 